\title{
Opportunistic Energy-Aware Amplify-and-Forward Cooperative Systems with Imperfect CSI
}

\author{
Osama Amin, Senior Member, IEEE, Ebrahim Bedeer, Member, IEEE, Mohamed H. Ahmed, \\ Senior Member, IEEE, Octavia A. Dobre, Senior Member, IEEE and Mohamed-Slim Alouini, Fellow, IEEE
}

\begin{abstract}
Recently, much attention has been paid to the green design of wireless communication systems using energy efficiency (EE) metrics that should capture all energy consumption sources to deliver the required data. In this paper, we design an energyefficient relay assisted communication system based on estimated channel state information (CSI). It employs amplify-andforward relaying and switches between different communication schemes, which are known as direct-transmission, two-hop and cooperative-transmission schemes, using the estimated CSI in order to maximize the EE. Two estimation strategies are assumed, namely disintegrated channel estimation and cascaded channel estimation. To formulate an accurate $\mathrm{EE}$ metric for the proposed opportunistic amplify-and-forward system, the channel estimation cost is reflected on the EE metric by including its impact in the signal-to-noise ratio term and in the energy consumption during the channels estimation phase. Based on the formulated EE metric, we propose an adaptive power allocation algorithm to maximize the EE of the proposed opportunistic amplify-andforward system with channel estimation. Furthermore, we study the impact of the estimation parameters on the proposed system via simulation examples.
\end{abstract}

Index Terms-Energy efficiency, cooperative communications, amplify-and-forward, estimated CSI, power allocation, opportunistic relaying.

\section{INTRODUCTION}

The rapid advances in wireless communications technology increase the widespread of wireless services and applications in the daily life. To further enhance the network capacity and obtain ubiquitous service, most of the research efforts focus on improving spectral efficiency and expanding the service coverage. Recently, the energy demand has increased significantly, which leads to a dramatic increase of the information and communication technology $\mathrm{CO}_{2}$ footprint in the environment, comparable to that of the global aviation industry [1]. Furthermore, the wireless devices lifetime has to be prolonged to meet the increased communication networks data traffic requirements. These serious challenges motivate

Copyright (c) 2015 IEEE. Personal use of this material is permitted. However, permission to use this material for any other purposes must be obtained from the IEEE by sending a request to pubs-permissions@ieee.org.

Osama Amin and Mohamed-S. Alouini are with with the Computer Electrical, and Mathematical Science and Engineering (CEMSE) Division, King Abdullah University of Science and Technology (KAUST), Thuwal, Makkah Province, Kingdom of Saudi Arabia, E-mail: \{osama.amin, slim.alouini\}@kaust.edu.sa.

Ebrahim Bedeer is with Faculty of Applied Science, University of British Columbia, Okanagan Campus, Kelowna, BC, Canada, V1V 1V7, E-mail: ebrahim.bedeer-mohamed@ubc.ca.

Mohamed H. Ahmed and Octavia A. Dobre are with Faculty of Engineering and Applied Science, Memorial University of Newfoundland, St. John's, Canada, A1C 5S7, E-mail: \{mhahmed, odobre\}@mun.ca. engineers to steer the attention towards the energy-aware design, which becomes a global design goal in both academic and industrial communities [2], [3]. For this purpose, the energy efficiency (EE) metric is adopted to capture all energy consumption sources used in the communication scenario to deliver the required data successfully [4].

Cooperative communications represent a powerful technology that provides reliable transmission by increasing the throughput, expanding the coverage and mitigating the fading without the need of multiple antennas [5]. Cooperative schemes create a virtual distributed antenna system using other available nodes in the network, i.e., the relays. Therefore, single-antenna communication nodes with limited-power capability can achieve long-distance reliable communications by using cooperative schemes with a reduced cost. As a result, cooperative communications are expected to participate effectively towards green communications [6].

Recently, the EE of cooperative communications has been investigated and a general framework for the energy-efficient relaying techniques is discussed in [6]. The energy-efficient resource allocation problem is investigated for the decode-andforward (DF) and for the amplify-and-forward (AF) [7]-[14].

Analyzing and designing communication system with imperfect channel state information (CSI) received a lot of attention recently [15], [16]. The energy aware design for the AF relay mode is employed considering only perfect channel state information (CSI) in [7]-[11]. In [7], Waqar et al. investigated the problem of multihop AF relaying without the availability of direct link and allocate the available resources to maximize the EE for a fixed bit error rate. In [8], Huang et $a l$. studied the EE and spectral efficiency trade-off for single relay cooperative scheme by optimizing the $\mathrm{EE}$ and imposing constraints on spectral efficiency and transmitted power. In [9], Sun et al. considered an EE-oriented design of the two source communication problem via a single relay. In [10], Cheung et al. solved the EE-based resource allocation problem of cellular downlink system with fixed relaying nodes. On the other hand, Loodaricheh et al. let the users play the relaying role in the same scenario and allocate the available resources to maximize the EE while guaranteeing minimum quality of service requirements [11].

The channel estimation impact on cooperative communications has been investigated for single and multiple relays with one and two-ways communications [17], [18]. Designing energy-efficient AF relaying systems based on imperfect or limited channel state information (CSI) is a challenging and interesting problem [12]-[14]. In [12], Zappone et al. 
explored the power allocation problem of two-hop multipleinput multiple-output AF cooperative system using statistical CSI. The same authors extended the problem to include the direct link and computed the source and relay power allocation in addition to the optimal conditions of beamforming that maximize the EE in [13]. In [14], Le et al. assumed a twohop AF relaying for the downlink multiuser communications, which uses one bit feedback. The authors considered a fixed data rate for multiple users, where the suggested centralized and distributed strategies notify the base station if the received signal-to-noise ratio (SNR) is below a certain threshold.

The aforementioned works in [12]-[14] designed the energy-efficient cooperative AF systems based on perfect instantaneous knowledge of CSI, or average CSI. In this paper, we design a relay-assisted system that uses estimated CSI and maximizes the EE. The main contributions of this paper can be summarized as follows:

- Based on estimated CSI, we first propose an opportunistic AF (OAF) system that switches between direct transmission (DT), two-hop (TH) and cooperative transmission (CT) schemes to achieve maximum EE.

- We formulate the EE metric to capture the channel estimation cost based on two channel estimation strategies, namely disintegrated channel estimation (DCE) and cascaded channel estimation (CCE). The channel estimation cost is reflected on the SNR reduction that results from the estimation process and the consumed energy in the channels estimation phase.

- We propose an adaptive OAF (AOAF) system that allocates the power to the source and relay to maximize the EE of the OAF system. Towards this end, we introduce three power allocation algorithms to compute the power for both source and relay nodes for different transmission schemes and the proposed algorithm chooses the appropriate one based on the maximum achievable EE.

- We investigate through simulation results:

- The effectiveness of the power allocation for the OAF system compared with equal power allocation;

- The importance of including the channel estimation cost on the EE metric for the AOAF system design;

- The EE benefits of adopting either estimation strategy, i.e., CCE or DCE;

- The dominant scheme of OAF;

- The impact of relay location;

- The impact of channel estimation quality.

The rest of the paper is organized as follows: In Section II, we introduce the system model with the two adopted relayassisted channel estimation strategies, respectively. In Section III, we formulate the EE of the relay-assisted OAF system with imperfect CSI. In Section IV, we derive the energy-efficient power allocation for the proposed system. In Section V, we present a comprehensive simulation study to illustrate the performance of the proposed relaying system. Finally, Section VI concludes the paper.

Notation: $(.)^{\mathrm{T}},(.)^{*}$, and $|$.$| denote transpose, conjugate, and$ absolute value operations, respectively. $\mathbb{E}$ denotes expectation. Bold upper-case letters denote the matrices and bold lower- case letters denote the vectors. $\mathbf{I}_{N}$ is an identity matrix with size $N$.

\section{SYSTEM MODEL}

We consider a communication system consisting of a source node $(S)$, a destination node $(D)$, and a half-duplex relay node $(R)$, that can be used to improve the system performance though cooperative techniques. The source node sends its data to the destination node using one of three possible transmission schemes: 1) direct transmission (DT), 2) twohop (TH) relaying, and 2) cooperative transmission (CT). The relay node is assumed to work in the AF mode through two orthogonal transmission phases, called broadcasting and relaying. In the first phase, $S$ broadcasts its data to be received by $R$ and possibly by $D$, and in the second phase, $S$ remains silent, while $R$ transmits an amplified version of the signal (after proper normalization) to $D$. Prior to the two phases of data transmission, a sequence of pilots, as shown in Fig. 1, is sent via the communication links to estimate the channels so that the optimal transmission scheme is chosen and the power of both $S$ and $R$ can be adapted to maximize the EE. We assume quasi-static block fading channel, where the channel is constant over the data and pilot blocks, i.e., both blocks are assumed to be within the coherence time of the channel. The energy-efficient scheme is chosen at $D$ and both $S$ and $R$ are informed via a feedback channel. Then, $m$ data packets are sent if TH or CT schemes are chosen, while $2 m$ data packets are sent if DT scheme is chosen.

The communication channels $h_{\mathrm{XY}}, \mathrm{X}, \mathrm{Y} \in\{\mathrm{S}, \mathrm{R}, \mathrm{D}\}$, are modelled as complex Gaussian distributed random variables with zero mean and variance $\sigma_{h_{\mathrm{XY}}}^{2}$, and its estimate is defined as $\hat{h}_{\mathrm{XY}}$. The large scale power coefficient that represents the path loss is modelled as $G_{\mathrm{XY}}=\left(4 \pi d_{0} / \lambda\right)^{-2}\left(d_{\mathrm{XY}} / d_{0}\right)^{-\nu}$ [19], where $d_{\mathrm{XY}}$ is the distance between different nodes, $d_{0}$ is the reference distance, $\lambda$ is the wavelength and $\nu$ is the path loss exponent. For channel estimation, we consider two strategies: DCE and CCE that use linear minimum mean square error (LMMSE) estimator [20]. We employ these two strategies according to the availability of the channel estimator at $R$. Based on the estimated CSI, which is assumed to be available at $D$ [20], the OAF system selects one of the aforementioned schemes, i.e., DT, TH or CT, according to the maximum EE. In addition, further EE improvement can be obtained by optimizing the power of both $S$ and $R$ (in case of $\mathrm{TH}$ or $\mathrm{CT}$ ) as it will be discussed in the next sections.

\section{A. Disintegrated Channel Estimation}

The DCE strategy can be adopted only when the relay is equipped with a channel estimator. Adopting the DCE strategy enables the relay to implement instantaneous power scaling, which leads to a better error probability performance than the relay that uses average power scaling [20], [21]. As such, the channel estimation occurs in two phases, broadcast channel estimation phase and relaying channel estimation phase. After the first estimation phase, the relay estimates $h_{\mathrm{SR}}$ and the destination estimates $h_{\mathrm{SD}}$ assuming known channels statistical information $\sigma_{h_{\mathrm{SR}}}^{2}$ and $\sigma_{h_{\mathrm{SD}}}^{2}$, and for a given path 


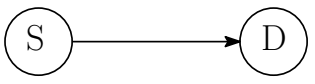

EstimationEstimation

\begin{tabular}{|l|l|l|l|l|l|l|l|} 
Phase 1 & Phase 2 & $d_{1}$ & $d_{2}$ & $d_{3}$ & $d_{4}$ & $\cdots$ & $\ldots \ldots \ldots$ \\
\hline
\end{tabular}

Data Transmission

a) DT-scheme

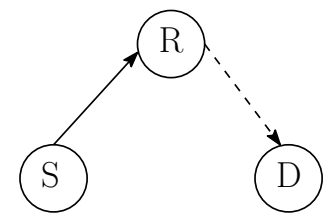

EstimationEstimatio Phase 1 Phase 2

Data Transmission

b) TH-scheme

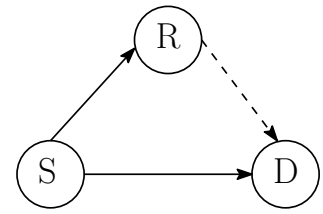

Estimation Estimation $\quad$ Data Transmission

\begin{tabular}{|l|l|l|l|l|l|l|l|}
\hline Phase 1 & Phase 2 & $d_{1}$ & & $d_{2}$ & & $\ldots \ldots \ldots \ldots$ & $d_{m}$ \\
\hline
\end{tabular}

c) CT-scheme

$\longrightarrow \quad$ Broadcasting phase

$-\ldots$ Relaying phase

Fig. 1. Adopted transmission schemes in the OAF system.

loss conditions $G_{\mathrm{SR}}$ and $G_{\mathrm{SD}}$. The mean-square-error (MSE) for $\hat{h}_{\mathrm{SR}}$ and $\hat{h}_{\mathrm{SD}}$ are expressed as [20], [22],

$$
\begin{gathered}
\sigma_{\mathrm{e}, \mathrm{SR}}^{2}=\frac{\sigma_{h_{\mathrm{SR}}}^{2} \sigma_{n}^{2}}{\sigma_{n}^{2}+N G_{\mathrm{SR}} P_{\mathrm{P}, \mathrm{S}} \sigma_{h_{\mathrm{SR}}}^{2}}, \\
\sigma_{\mathrm{e}, \mathrm{SD}}^{2}=\frac{\sigma_{h_{\mathrm{SD}}}^{2} \sigma_{n}^{2}}{\sigma_{n}^{2}+N G_{\mathrm{SD}} P_{\mathrm{P}, \mathrm{S}} \sigma_{h_{\mathrm{SD}}}^{2}},
\end{gathered}
$$

where $P_{\mathrm{P}, \mathrm{S}}$ is the power of the transmitted pilot from the source, and $\sigma_{n}^{2}$ is the noise variance, which is computed from $\sigma_{n}^{2}=N_{0} N_{\mathrm{f}} B$, where $N_{0}$ is the thermal noise power spectral density, $N_{\mathrm{f}}$ is the receiver noise figure and $B$ is the transmission bandwidth.

In the relaying estimation phase, the relay sends new $N$ pilots to the destination to estimate the $h_{\mathrm{RD}}$ channel with MSE [20]

$$
\sigma_{\mathrm{e}, \mathrm{RD}}^{2}=\frac{\sigma_{h_{\mathrm{RD}}}^{2} \sigma_{n}^{2}}{\sigma_{n}^{2}+N G_{\mathrm{RD}} P_{\mathrm{P}, \mathrm{R}} \sigma_{h_{\mathrm{RD}}}^{2}},
$$

where $P_{\mathrm{P}, \mathrm{R}}$ is the power of the transmitted pilot from the source.

The cooperative channel link, i.e., $S \rightarrow R \rightarrow D$, is estimated to be $\hat{h}_{\mathrm{SR}} \hat{h}_{\mathrm{RD}}$ with MSE $\sigma_{\mathrm{e}, \mathrm{DCE}}^{2}$, which is expressed based on [20] as

$$
\begin{aligned}
\sigma_{\mathrm{e}, \mathrm{DCE}}^{2}= & \sigma_{\mathrm{e}, \mathrm{SR}}^{2} \sigma_{\mathrm{e}, \mathrm{RD}}^{2} \\
& \times\left(1+\frac{N G_{\mathrm{RD}} P_{\mathrm{P}, \mathrm{R}} \sigma_{h_{\mathrm{RD}}}^{2}}{\sigma_{n}^{2}}+\frac{N G_{\mathrm{SR}} P_{\mathrm{P}, \mathrm{S}} \sigma_{h_{\mathrm{SR}}}^{2}}{\sigma_{n}^{2}}\right),
\end{aligned}
$$

\section{B. Cascaded Channel Estimation}

The CCE strategy is suitable for a relay that is not equipped with channel estimator. Hence, the relay can only use average power scaling, which results in inferior error probability performance when compared with the cooperative system with instantaneous power scaling [20], [21]. This estimation strategy is implemented also in two phases, broadcast and relaying phases, but the estimation of the cooperative link is done at the destination terminal. In the first transmission phase, the relay receives the pilot(s) signal and normalizes it by the average received power before amplifying it with power equal to $P_{\mathrm{P}, \mathrm{R}}$. The channel estimation MSE for the $S \rightarrow R \rightarrow D$ link for the CCE strategy is expressed based on [20] as

$$
\sigma_{\mathrm{e}, \mathrm{CCE}}^{2}=\frac{\sigma_{h_{\mathrm{SR}}}^{2} \sigma_{h_{\mathrm{RD}}}^{2} \sigma_{n}^{2}}{\sigma_{n}^{2}+N G_{\mathrm{SRD}} P_{\mathrm{P}, \mathrm{S}} P_{\mathrm{P}, \mathrm{R}} \sigma_{h_{\mathrm{SR}}}^{2} \sigma_{h_{\mathrm{RD}}}^{2}},
$$

where $G_{\mathrm{SRD}}$ is defined as

$$
G_{\mathrm{SRD}}=\frac{G_{\mathrm{SR}} G_{\mathrm{RD}}}{G_{\mathrm{SR}} P_{\mathrm{P}, \mathrm{S}} \sigma_{h_{\mathrm{SR}}}^{2}+G_{\mathrm{RD}} P_{\mathrm{P}, \mathrm{R}} \sigma_{h_{\mathrm{RD}}}^{2}+\sigma_{n}^{2}} .
$$

\section{ENERGY EFFICIENCY OF THE OPPORTUNISTIC AMPLIFY-AND-FORWARD SYSTEM}

In this section, we formulate the $\mathrm{EE}$ metric for the $\mathrm{OAF}$ system by taking into account the channel estimation cost. This cost appears as a reduction in the SNR that results from the estimation error and an increase in the energy consumption as a result of pilot(s) transmission and reception. The proposed system switches between the three possible schemes: DT, TH and CT according to the maximum EE. Thus, the EE of the proposed system is expressed as

$$
\eta_{\mathrm{OAF}}=\max \left(\eta_{\mathrm{DT}}, \eta_{\mathrm{TH}}, \eta_{\mathrm{CT}}\right),
$$

where $\eta_{\mathrm{DT}}, \eta_{\mathrm{TH}}$ and $\eta_{\mathrm{CT}}$ are the EE of the DT, TH, CT schemes, respectively; these schemes are illustrated in Figure 1. The EE of any scheme is defined as

$$
\eta=\frac{\mathcal{S}}{P_{\mathrm{T}}} \quad \text { bit } / \mathrm{Hz} / \text { Joule, }
$$

where $\mathcal{S}$ is the spectral efficiency and $P_{\mathrm{T}}$ is the total power consumption of all participated nodes during transmission and reception of the data and pilots. Before deciding on the transmission scheme, the CSI of the cooperative links is estimated by either DCE or CCE strategies, and assumed to be available at $D$ [20]. Then, the best EE scheme is selected based on (7) at $D$. The power consumption cost used for channels estimation is the same for the three schemes.

\section{A. Direct-Transmission Scheme}

When the DT scheme is selected to transmit the data, $R$ stays silent during the data transmission. The spectral efficiency ${ }^{1}$ of the adopted scheme is expressed in terms of the source power, the estimated CSI, and the MSE of the estimator as

$$
\mathcal{S}_{\mathrm{DT}}=\log _{2}\left(1+\frac{\gamma_{\mathrm{SD}} P_{\mathrm{S}}}{\varepsilon_{\mathrm{SD}} P_{\mathrm{S}}+1}\right) \quad \mathrm{bit} / \mathrm{s} / \mathrm{Hz}
$$

${ }^{1}$ The SE expressions used in the paper are lower bounds, which are found to be tight for Gaussian inputs [23], [24]. 
where $\gamma_{\mathrm{SD}}$ is the channel-to-noise ratio (CNR) defined as $\gamma_{\mathrm{SD}}=\left|\hat{h}_{\mathrm{SD}}\right|^{2} G_{\mathrm{SD}} / \sigma_{n}^{2}$, and $\varepsilon_{\mathrm{SD}}$ is the MSE-to-noise ratio defined as $\varepsilon_{\mathrm{SD}}=\sigma_{\mathrm{e}, \mathrm{SD}}^{2} G_{\mathrm{SD}} / \sigma_{n}^{2}$. $\varepsilon_{\mathrm{SD}}$ depends on deterministic parameters that are assumed to be available at $D$. The channel estimation footprint appears as a reduction of the signal-tonoise ratio through the $\varepsilon_{\mathrm{SD}}$ term in addition to affecting the signal power by $\left|\hat{h}_{\mathrm{SD}}\right|^{2}$. For a given channel statistical characteristics, certain communication range, and estimator specifications, i.e., number of pilots and its power, $\varepsilon_{\mathrm{SD}}$ is constant for any channel realization, while $\gamma_{\mathrm{SD}}$ changes according to the CSI. The total dissipated power ${ }^{2}$ when the DT scheme is selected for transmission is computed from

$$
P_{\mathrm{T}, \mathrm{DT}}=\kappa_{\mathrm{S}} P_{\mathrm{S}}+P_{\mathrm{c}, \mathrm{DT}}+P_{\mathrm{CE}, \mathrm{DT}}
$$

where $\kappa_{\mathrm{S}}$ is a power amplifier constant of the source node that depends on the power amplifier efficiency and peak-to-average power ratio, $P_{\mathrm{S}}$ is the source transmitted power, $P_{\mathrm{c}, \mathrm{DT}}$ is the circuits' power consumption cost of data transmission, and $P_{\mathrm{CE}, \mathrm{DT}}$ is the power consumption for channels estimation. $P_{\mathrm{c}, \mathrm{DT}}$ does not include the power consumption of the relay because it is turned off during the data transmission and is expressed as

$$
P_{\mathrm{c}, \mathrm{DT}}=P_{\mathrm{ct}, \mathrm{S}}+P_{\mathrm{cr}, \mathrm{D}}
$$

where $P_{\mathrm{ct}, \mathrm{S}}$ is the circuits' power consumption used for the transmission at the source node and $P_{\mathrm{cr}, \mathrm{D}}$ is the circuits' power consumption used to receive the data at the destination node. On the other hand, $P_{\mathrm{CE}, \mathrm{DT}}$ includes the power consumption of all nodes because all links have to be estimated before selecting the best scheme, and is determined from

$$
\begin{aligned}
& P_{\mathrm{CE}, \mathrm{DT}}=\frac{\rho_{\mathrm{DT}}}{2} \times \\
& \left(\kappa_{\mathrm{S}} P_{\mathrm{P}, \mathrm{S}}+\kappa_{\mathrm{R}} P_{\mathrm{P}, \mathrm{R}}+P_{\mathrm{ct}, \mathrm{S}}+P_{\mathrm{cr}, \mathrm{R}}+P_{\mathrm{ct}, \mathrm{R}}+2 P_{\mathrm{cr}, \mathrm{D}}\right),
\end{aligned}
$$

where $\kappa_{\mathrm{R}}$ is a power amplifier constant of the relay node, $\rho_{\mathrm{DT}}$ represents the pilots-to-data symbols ratio in the transmitted packet for the DT scheme, and $P_{\mathrm{ct}, \mathrm{R}}$ and $P_{\mathrm{cr}, \mathrm{R}}$ are the circuits' power consumption used for the transmission and reception of $R$, respectively.

\section{B. Two-Hop Scheme}

The TH scheme aims to use $R$ to deliver the data through AF relaying without the help of the direct link; thus, $D$ works only half of the transmission time and switches off during the other half. The spectral efficiency of the two-hop AF relaying system is written in terms of the source and relay power, $P_{\mathrm{S}}$ and $P_{\mathrm{R}}$, respectively, as

$$
\begin{array}{r}
\mathcal{S}_{\mathrm{TH}}=\frac{1}{2} \log _{2}\left(1+\frac{\gamma_{\mathrm{SRD}} P_{\mathrm{S}} P_{\mathrm{R}}}{\varepsilon_{\mathrm{SRD}} P_{\mathrm{S}} P_{\mathrm{R}}+\alpha_{\mathrm{SR}} P_{\mathrm{S}}+\alpha_{\mathrm{RD}} P_{\mathrm{R}}+1}\right) \\
\text { bit } / \mathrm{s} / \mathrm{Hz}, \quad(13)
\end{array}
$$

\footnotetext{
${ }^{2}$ Signal processing power is neglected with respect to other power terms [6], [25].
}

where $\gamma_{\mathrm{SRD}}$ is the CNR of the cooperative link, i.e., $S \rightarrow$ $R \rightarrow D$ link, and is expressed as

$$
\gamma_{\mathrm{SRD}}=\left\{\begin{array}{ll}
\frac{G_{\mathrm{SR}} G_{\mathrm{RD}}\left|\hat{h}_{\mathrm{SR}}\right|^{2}\left|\hat{h}_{\mathrm{RD}}\right|^{2}}{\sigma_{n}^{4}} & \text { for DCE } \\
\frac{G_{\mathrm{SR}} G_{\mathrm{RD}}\left|\hat{h}_{\mathrm{SRD}}\right|^{2}}{\sigma_{n}^{4}} & \text { for CCE }
\end{array},\right.
$$

$\alpha_{\mathrm{SR}}$ is the CNR of the $S \rightarrow R$ link and is expressed as

$$
\alpha_{\mathrm{SR}}=\left\{\begin{array}{cc}
\frac{G_{\mathrm{SR}}\left|\hat{h}_{\mathrm{SR}}\right|^{2}}{\sigma_{n}^{2}} & \text { for DCE } \\
\frac{G_{\mathrm{SR}} \sigma_{h_{\mathrm{SR}}^{2}}}{\sigma_{n}^{2}} & \text { for CCE }
\end{array}\right.
$$

$\alpha_{\mathrm{RD}}$ is the CNR of the $R \rightarrow D$ link and is expressed as

$$
\alpha_{\mathrm{RD}}=\left\{\begin{array}{ll}
\frac{G_{\mathrm{RD}}\left|\hat{h}_{\mathrm{RD}}\right|^{2}}{\sigma_{n}^{2}} & \text { for DCE } \\
\frac{G_{\mathrm{RD}} \sigma_{h_{\mathrm{RD}}}^{2}}{\sigma_{n}^{2}} & \text { for CCE }
\end{array},\right.
$$

and $\varepsilon_{\mathrm{SRD}}$ is the estimation MSE-to-noise ratio and is expressed as

$$
\varepsilon_{\mathrm{SRD}}=\left\{\begin{array}{ll}
\frac{G_{\mathrm{SR}} G_{\mathrm{RD}} \sigma_{\mathrm{e}, \mathrm{DCE}}^{2}}{\sigma_{n}^{4}} & \text { for DCE } \\
\frac{G_{\mathrm{SR}} G_{\mathrm{RD}} \sigma_{\mathrm{e}, \mathrm{CCE}}^{2}}{\sigma_{n}^{4}} & \text { for } \mathrm{CCE}
\end{array} .\right.
$$

$\varepsilon_{\mathrm{SRD}}$ is a deterministic parameter and is assumed to be known at $D$. The spectral efficiency of the TH scheme suffers from a loss factor of " $1 / 2$ " due to the usage of two time slots to deliver a symbol. We observe that the unavailability of the individual link channel estimates in CCE forces us to use the expected values of the channel gain instead of the actual value [20], as can be seen in (15) and (16).

The total power consumed during the TH AF communication scheme is computed from

$$
P_{\mathrm{T}, \mathrm{TH}}=\frac{1}{2}\left(\kappa_{\mathrm{S}} P_{\mathrm{S}}+\kappa_{\mathrm{R}} P_{\mathrm{R}}\right)+P_{\mathrm{c}, \mathrm{TH}}+P_{\mathrm{CE}},
$$

where $P_{\mathrm{CE}}$ is the power consumption due to channel estimation when $\mathrm{TH}$ or $\mathrm{CT}$ is adopted, and $P_{\mathrm{c}}$ is the power consumed in all circuits, which can be expressed as

$$
P_{\mathrm{c}, \mathrm{TH}}=\frac{1}{2}\left(P_{\mathrm{ct}, \mathrm{S}}+P_{\mathrm{cr}, \mathrm{R}}+P_{\mathrm{ct}, \mathrm{R}}+P_{\mathrm{cr}, \mathrm{D}}\right) .
$$

$P_{\mathrm{CE}}$ is expressed as

$$
\begin{aligned}
& P_{\mathrm{CE}}=\frac{\rho}{2} \times \\
& \left(\kappa_{\mathrm{S}} P_{\mathrm{P}, \mathrm{S}}+\kappa_{\mathrm{R}} P_{\mathrm{P}, \mathrm{R}}+P_{\mathrm{ct}, \mathrm{S}}+P_{\mathrm{cr}, \mathrm{R}}+P_{\mathrm{ct}, \mathrm{R}}+2 P_{\mathrm{cr}, \mathrm{D}}\right),
\end{aligned}
$$

where $\rho=2 \rho_{\mathrm{DT}}$ is the pilots-to-data symbols ratio in the transmitted packet for both schemes TH and CT. All power terms in (18), (19) and (20) have the "1/2" factor because the circuits of $S$ and $R$ work only half of the transmission period with the exception of $D$ during the estimation phases, where its received circuits work to receive the pilots from the direct link in the first phase and from the cooperative link in the second phase. 


\section{Cooperative-Transmission Scheme}

In the CT scheme, $D$ works the whole transmission period and receives the data from $S$ and $R$ during the two transmission phases. The spectral efficiency of the two-hop AF relaying system is determined from

$$
\begin{aligned}
\mathcal{S}_{\mathrm{CT}} & =\frac{1}{2} \log _{2}\left(1+\frac{\gamma_{\mathrm{SD}} P_{\mathrm{S}}}{\varepsilon_{\mathrm{SD}} P_{\mathrm{S}}+1}\right. \\
+ & \left.\frac{\gamma_{\mathrm{SRD}} P_{\mathrm{S}} P_{\mathrm{R}}}{\varepsilon_{\mathrm{SRD}} P_{\mathrm{S}} P_{\mathrm{R}}+\alpha_{\mathrm{SR}} P_{\mathrm{S}}+\alpha_{\mathrm{RD}} P_{\mathrm{R}}+1}\right) \mathrm{bit} / \mathrm{s} / \mathrm{Hz}
\end{aligned}
$$

Similar to the TH scheme, the CT scheme needs two transmission phases to deliver the data, thus it suffers from a rate loss by factor of " $1 / 2$ " as can be seen in (21). The total power consumed during the CT scheme is given by

$$
P_{\mathrm{T}, \mathrm{CT}}=\frac{1}{2}\left(\kappa_{\mathrm{S}} P_{\mathrm{S}}+\kappa_{\mathrm{R}} P_{\mathrm{R}}\right)+P_{\mathrm{c}, \mathrm{CT}}+P_{\mathrm{CE}},
$$

where $P_{\mathrm{c}, \mathrm{CT}}$ is the power consumed in all circuits, which can be expressed as

$$
P_{\mathrm{c}, \mathrm{CT}}=\frac{1}{2}\left(P_{\mathrm{ct}, \mathrm{S}}+P_{\mathrm{cr}, \mathrm{R}}+P_{\mathrm{ct}, \mathrm{R}}+2 P_{\mathrm{cr}, \mathrm{D}}\right) \text {. }
$$

Similar to the TH scheme, the power consumption of the $S$ and $R$ nodes is factored by " $1 / 2$ " because the circuits work during one of the two transmission phases, while for the $D$ node, they work during both transmission phases. We observe from the EE expression in (8) and related equations that improving the channel estimation via either increasing the pilots' power or the number of pilots can improve significantly the SNR, but it does not necessarily improve the EE. In addition, we need to adjust the data power allocated to $S$ and $R$ to maximize the EE for a required estimation quality. In the following section, we focus on this problem and propose three algorithms that maximize the EE for the three transmission schemes and choose the best scheme that achieves the maximum EE for both CCE and DCE strategies.

\section{Adaptive Power Allocation for the OPPORTUNISTIC AMPLIFY-AND-FORWARD SYSTEM}

In this section, we propose the AOAF system that assumes fixed known channel estimation parameters represented by the number of pilots, pilot(s) power and pilot(s) density with respect to the data, and allocates the data power to both $S$ and $R$ to maximize the EE of OAF. The EE of the AOAF system is found from

$$
\eta_{\mathrm{OAF}}^{*}=\max \left(\eta_{\mathrm{DT}}^{*}, \eta_{\mathrm{TH}}^{*}, \eta_{\mathrm{CT}}^{*}\right),
$$

where $\eta_{\mathrm{DT}}^{*}, \eta_{\mathrm{TH}}^{*}$ and $\eta_{\mathrm{CT}}^{*}$ are the maximized EE of the DT, $\mathrm{TH}$, and CT schemes, respectively. The node $D$ performs the selection process based on the estimated CSI of the communication links assuming the availability of other fixed system parameters. Then, $D$ acknowledges both $S$ and $R$ with the selected scheme along with the transmission power using a feedback channel. The power allocation of the maximized EE systems is computed as described in the following subsections.

\section{A. Energy-Aware Power Allocation for Direct-Transmission Scheme}

The power allocation problem of the DT scheme is expressed as

$$
\begin{array}{cl}
\max _{P_{\mathrm{S}}} & \eta_{\mathrm{DT}}=\frac{\log _{2}\left(1+\frac{\gamma_{\mathrm{SD}} P_{\mathrm{S}}}{\varepsilon_{\mathrm{SD}} P_{\mathrm{S}}+1}\right)}{\kappa_{\mathrm{S}} P_{\mathrm{S}}+P_{\mathrm{c}, \mathrm{DT}}+P_{\mathrm{CE}, \mathrm{DT}}} \\
\text { subject to } & 0<P_{\mathrm{S}} \leq P_{\mathrm{S}, \max },
\end{array}
$$

where $P_{\mathrm{S}, \max }$ is the maximum power budget of $S$. The objective function in (25) is a pseudo-concave function in $P_{\mathrm{S}}$ where it is a ratio of a concave $\mathrm{e}^{3}$ and a positive linear functions [26]. One way to solve this problem is to use the Dinkelbach method [27], which converts the fractional pseudoconcave objective function into a concave function. Hence, we obtain the following equivalent optimization problem

$$
\begin{array}{cl}
\max _{P_{\mathrm{S}}} & f_{\mathrm{DT}}\left(P_{\mathrm{S}}\right) \\
\text { subject to } & 0<P_{\mathrm{S}} \leq P_{\mathrm{S}, \max },
\end{array}
$$

where the objective function $f_{\mathrm{DT}}\left(P_{\mathrm{S}}\right)$ is defined as

$$
\begin{aligned}
f_{\mathrm{DT}}\left(P_{\mathrm{S}}\right)=\log _{2} & \left(1+\frac{\gamma_{\mathrm{SD}} P_{\mathrm{S}}}{\varepsilon_{\mathrm{SD}} P_{\mathrm{S}}+1}\right) \\
& -q_{\mathrm{DT}}\left(\kappa_{\mathrm{S}} P_{\mathrm{S}}+P_{\mathrm{c}, \mathrm{DT}}+P_{\mathrm{CE}, \mathrm{DT}}\right),
\end{aligned}
$$

with $q_{\mathrm{DT}}$ as a constant that is equal to the maximum EE value of DT scheme, i.e., $q_{\mathrm{DT}}=\max \eta_{\mathrm{DT}}$. In other words, if $P_{\mathrm{S}}^{*}=$ $\arg \max \eta_{\mathrm{DT}}$, then $f_{\mathrm{DT}}\left(P_{\mathrm{S}}^{*}\right)=0$ [27]. Define $\mathcal{G}_{\mathrm{DT}}\left(P_{\mathrm{S}}\right)$ as

$$
\mathcal{G}_{\mathrm{DT}}\left(P_{\mathrm{S}}\right)=1+\frac{\gamma_{\mathrm{SD}} P_{\mathrm{S}}}{\varepsilon_{\mathrm{SD}} P_{\mathrm{S}}+1} .
$$

The second derivative of $\mathcal{G}_{\mathrm{DT}}\left(P_{\mathrm{S}}\right)$ is expressed as

$$
\frac{\partial^{2} \mathcal{G}_{\mathrm{DT}}}{\partial P_{\mathrm{S}}^{2}}=-2 \frac{\varepsilon_{\mathrm{SD}} \gamma_{\mathrm{SD}}}{\left(\varepsilon_{\mathrm{SD}} P_{\mathrm{S}}+1\right)^{3}} .
$$

Since $\frac{\partial^{2} \mathcal{G}_{\mathrm{DT}}}{\partial P_{\mathrm{S}}^{2}}<0$, then $\mathcal{G}_{\mathrm{DT}}$ is concave in $P_{\mathrm{S}}$. By following the operations that preserve convexity/concavity [28], we can find that the logarithmic term in (27) is also a concave function, and thus $f_{\mathrm{DT}}\left(P_{\mathrm{S}}\right)$ is shown to be concave.

By differentiating $f_{\mathrm{DT}}\left(P_{\mathrm{S}}\right)$ with respect to $P_{\mathrm{S}}$, we obtain

$$
\begin{aligned}
& P_{\mathrm{S}}=\frac{1}{2}\left(\frac{2 \varepsilon_{\mathrm{SD}}+\gamma_{\mathrm{SD}}}{\varepsilon_{\mathrm{SD}}+\gamma_{\mathrm{SD}}}\right) \times \\
& {\left[\sqrt{1+4 \frac{\varepsilon_{\mathrm{SD}}+\gamma_{\mathrm{SD}}}{\left(2 \varepsilon_{\mathrm{SD}}+\gamma_{\mathrm{SD}}\right)^{2}}\left(\frac{\gamma_{\mathrm{SD}}}{q_{\mathrm{DT}} \kappa_{\mathrm{S}} \log 2}-1\right)}-1\right] .}
\end{aligned}
$$

The source power allocation solution, $P_{\mathrm{S}}^{*}$, can be computed from (30) based on the value of $q_{\mathrm{DT}}$, which can be computed iteratively based on the Dinkelback method [27], as shown in Algorithm I.

\footnotetext{
${ }^{3}$ The proof is provided in the following discussion.
} 


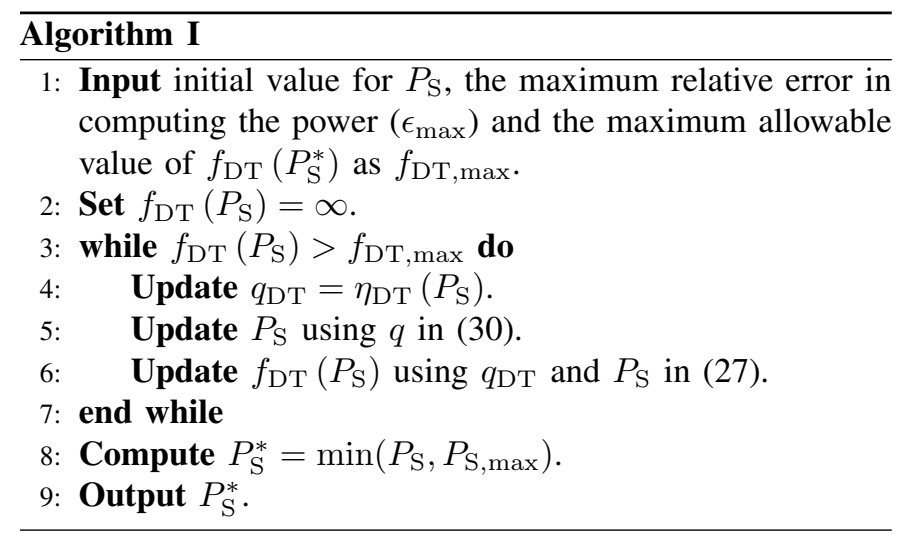

\section{B. Energy-Aware Power Allocation for Two-Hop Scheme}

The energy-efficient power allocation for TH scheme is formulated as

$$
\begin{array}{cl}
\max _{P_{\mathrm{S}}, P_{\mathrm{R}}} & \eta_{\mathrm{TH}}=\frac{\log _{2}\left(1+\frac{\gamma_{\mathrm{SRD}} P_{\mathrm{S}} P_{\mathrm{R}}}{\varepsilon_{\mathrm{SRD}} P_{\mathrm{S}} P_{\mathrm{R}}+\alpha_{\mathrm{SR}} P_{\mathrm{S}}+\alpha_{\mathrm{RD}} P_{\mathrm{R}}+1}\right)}{\left(\kappa_{\mathrm{S}} P_{\mathrm{S}}+\kappa_{\mathrm{R}} P_{\mathrm{R}}\right)+2 P_{\mathrm{c}, \mathrm{TH}}+2 P_{\mathrm{CE}}} \\
\text { subject to } & 0<P_{\mathrm{S}} \leq P_{\mathrm{S}, \max } \\
& 0<P_{\mathrm{R}} \leq P_{\mathrm{R}, \max },
\end{array}
$$

where $P_{\mathrm{R}, \max }$ is the maximum power budget of $R$. Similar to the DT scheme, we use the Dinkelbach method [27] to convert the optimization problem in (31) to the following equivalent optimization problem

$$
\begin{aligned}
\max _{P_{\mathrm{S}}, P_{\mathrm{R}}} & f_{\mathrm{TH}}\left(P_{\mathrm{S}}, P_{\mathrm{R}}\right) \\
\text { subject to } & 0<P_{\mathrm{S}} \leq P_{\mathrm{S}, \max } \\
& 0<P_{\mathrm{R}} \leq P_{\mathrm{R}, \max },
\end{aligned}
$$

where $f_{\mathrm{TH}}\left(P_{\mathrm{S}}, P_{\mathrm{R}}\right)$ is defined as

$f_{\mathrm{TH}}\left(P_{\mathrm{S}}, P_{\mathrm{R}}\right)=\log \left(1+\frac{\gamma_{\mathrm{SRD}} P_{\mathrm{S}} P_{\mathrm{R}}}{\varepsilon_{\mathrm{SRD}} P_{\mathrm{S}} P_{\mathrm{R}}+\alpha_{\mathrm{SR}} P_{\mathrm{S}}+\alpha_{\mathrm{RD}} P_{\mathrm{R}}+1}\right)$

$\times\left(\frac{1}{\log 2}\right)-q_{\mathrm{TH}}\left(\kappa_{\mathrm{S}} P_{\mathrm{S}}+\kappa_{\mathrm{R}} P_{\mathrm{R}}+P_{\mathrm{c}}\right)$,

and $q_{\mathrm{TH}}$ is a constant that equals the maximum $\mathrm{EE}$ value of the TH scheme, i.e., $q_{\mathrm{TH}}=\max \eta_{\mathrm{TH}}$. In other words, if $\left(P_{\mathrm{S}}^{*}, P_{\mathrm{R}}^{*}\right)=\arg \max \eta_{\mathrm{TH}}$, then $f_{\mathrm{TH}}\left(P_{\mathrm{S}}^{*}, P_{\mathrm{R}}{ }^{*}\right)=0$ [27]. The other parameters in (33) depends on the statistical characteristic of the channels and the estimation quality, which are assumed to be known. Define the argument of the logarithmic term in (33) as

$$
\mathcal{G}_{\mathrm{TH}}\left(P_{\mathrm{S}}, P_{\mathrm{R}}\right)=1+\frac{\gamma_{\mathrm{SRD}} P_{\mathrm{S}} P_{\mathrm{R}}}{\varepsilon_{\mathrm{SRD}} P_{\mathrm{S}} P_{\mathrm{R}}+\alpha_{\mathrm{SR}} P_{\mathrm{S}}+\alpha_{\mathrm{RD}} P_{\mathrm{R}}+1} .
$$

For a given value of $P_{\mathrm{R}}$, we find that

$$
\frac{\partial^{2} \mathcal{G}_{\mathrm{TH}}}{\partial P_{\mathrm{S}}^{2}}=-\frac{2 \gamma_{\mathrm{SRD}}\left(\alpha_{\mathrm{RD}} P_{\mathrm{R}}+1\right)\left(\varepsilon_{\mathrm{SRD}} P_{\mathrm{R}}+\alpha_{\mathrm{SR}}\right) P_{\mathrm{R}}}{\left(\varepsilon_{\mathrm{SRD}} P_{\mathrm{S}} P_{\mathrm{R}}+\alpha_{\mathrm{SR}} P_{\mathrm{S}}+\alpha_{\mathrm{RD}} P_{\mathrm{R}}+1\right)^{3}} \leq 0,
$$

which proves that $\mathcal{G}_{\mathrm{TH}}$ is concave in $P_{\mathrm{S}}$. As a result, the logarithmic term in (33) is also a concave function in $P_{\mathrm{S}}$, and
$f_{\mathrm{TH}}\left(P_{\mathrm{S}}, P_{\mathrm{R}}\right)$ is concave in $P_{\mathrm{S}}$. Then, if we assume fixed $P_{\mathrm{S}}$, we find that

$$
\frac{\partial^{2} \mathcal{G}_{\mathrm{TH}}}{\partial P_{\mathrm{R}}^{2}}=-\frac{2 \gamma_{\mathrm{SRD}}\left(\alpha_{\mathrm{SR}} P_{\mathrm{S}}+1\right)\left(\varepsilon_{\mathrm{SRD}} P_{\mathrm{S}}+\alpha_{\mathrm{RD}}\right) P_{\mathrm{S}}}{\left(\varepsilon_{\mathrm{SRD}} P_{\mathrm{S}} P_{\mathrm{R}}+\alpha_{\mathrm{SR}} P_{\mathrm{S}}+\alpha_{\mathrm{RD}} P_{\mathrm{R}}+1\right)^{3}} \leq 0
$$

that leads to concave characteristics of $\mathcal{G}_{\mathrm{TH}}$ in $P_{\mathrm{R}}$; accordingly, $f_{\mathrm{TH}}\left(P_{\mathrm{S}}, P_{\mathrm{R}}\right)$ is concave in $P_{\mathrm{R}}$.

By differentiating $f_{\mathrm{TH}}\left(P_{\mathrm{S}}, P_{\mathrm{R}}\right)$ with respect to $P_{\mathrm{S}}$ we obtain (37). Similarly, by differentiating $f_{\mathrm{TH}}\left(P_{\mathrm{S}}, P_{\mathrm{R}}\right)$ with respect to $P_{\mathrm{R}}$ we obtain (38).

Based on (37) and (38), we develop Algorithm II that uses the Dinklebach method and calculates iteratively the energyefficient power allocation solution for the $\mathrm{TH}$ scheme.

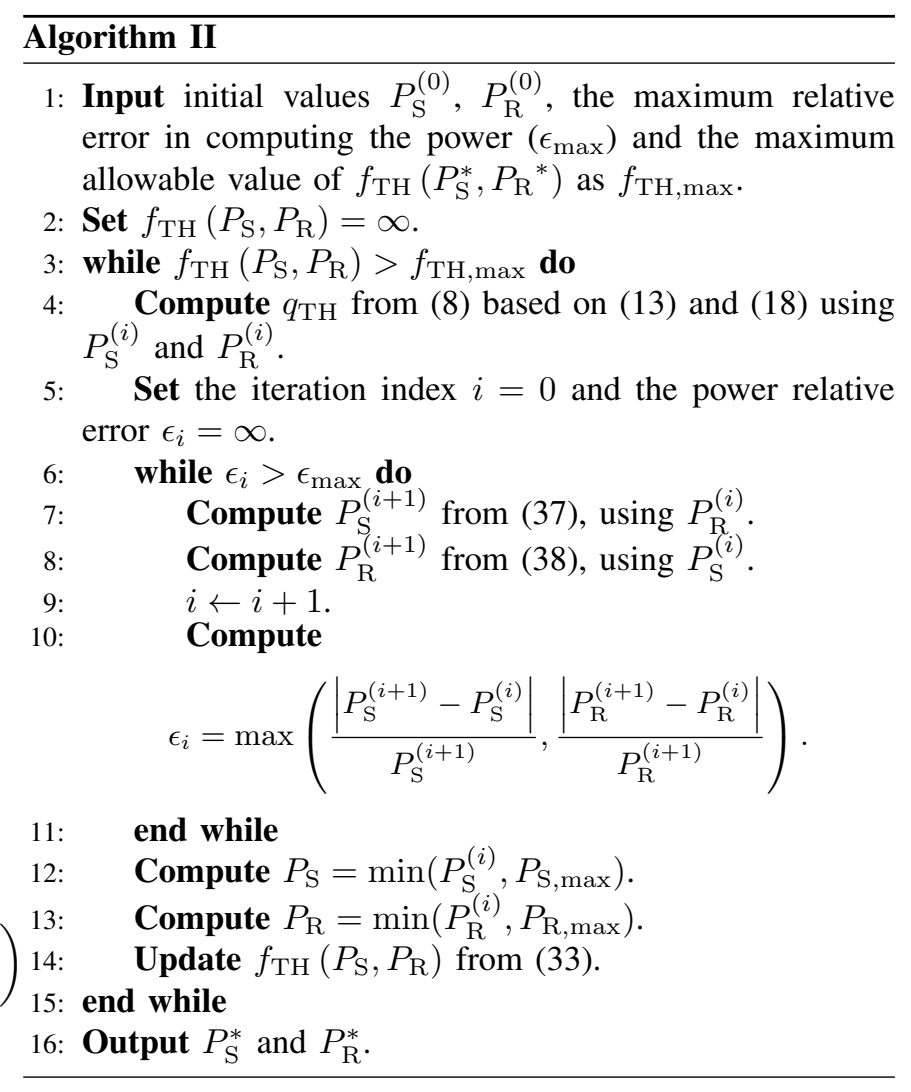

\section{Energy-Aware Power Allocation for Cooperative Transmis- sion Scheme}

The power allocation problem of the CT scheme is formulated as

$\max _{P_{\mathrm{S}}, P_{\mathrm{R}}} \eta_{\mathrm{CT}}=\frac{\log _{2}\left(1+\frac{\gamma_{\mathrm{SD}} P_{\mathrm{S}}}{\varepsilon_{\mathrm{SD}} P_{\mathrm{S}}+1}+\frac{\gamma_{\mathrm{SRD}} P_{\mathrm{S}} P_{\mathrm{R}}}{\varepsilon_{\mathrm{SRD}} P_{\mathrm{S}} P_{\mathrm{R}}+\alpha_{\mathrm{SR}} P_{\mathrm{S}}+\alpha_{\mathrm{RD}} P_{\mathrm{R}}+1}\right)}{\kappa_{\mathrm{S}} P_{\mathrm{S}}+\kappa_{\mathrm{R}} P_{\mathrm{R}}+2 P_{\mathrm{c}, \mathrm{CT}}+2 P_{\mathrm{CE}}}$

subject to $0<P_{\mathrm{S}} \leq P_{\mathrm{S}, \max }$

$0<P_{\mathrm{R}} \leq P_{\mathrm{R}, \max }$.

Similar to the DT and CT schemes, the optimization problem of the CT scheme in (39) is converted to the following equivalent optimization problem using the Dinkelbach method [27] 


$$
\begin{aligned}
P_{\mathrm{S}}=\frac{\left(\alpha_{\mathrm{RD}} P_{\mathrm{R}}+1\right)\left(\left(\left(2 \varepsilon_{\mathrm{SRD}}+\gamma_{\mathrm{SRD}}\right) P_{\mathrm{R}}+2 \alpha_{\mathrm{SR}}\right)\right)}{2\left(\varepsilon_{\mathrm{SRD}} P_{\mathrm{R}}+\alpha_{\mathrm{SR}}\right)\left(\left(\varepsilon_{\mathrm{SRD}}+\gamma_{\mathrm{SRD}}\right) P_{\mathrm{R}}+\alpha_{\mathrm{SR}}\right)} \times \\
\left(\sqrt{1+4 \frac{\left(\varepsilon_{\mathrm{SRD}} P_{\mathrm{R}}+\alpha_{\mathrm{SR}}\right)\left(\left(\varepsilon_{\mathrm{SRD}}+\gamma_{\mathrm{SRD}}\right) P_{\mathrm{R}}+\alpha_{\mathrm{SR}}\right)}{\left(\alpha_{\mathrm{RD}} P_{\mathrm{R}}+1\right)\left(\left(2 \varepsilon_{\mathrm{SRD}}+\gamma_{\mathrm{SRD}}\right) P_{\mathrm{R}}+2 \alpha_{\mathrm{SR}}\right)^{2}}\left(\frac{\gamma_{\mathrm{SRD}} P_{\mathrm{R}}}{q_{\mathrm{TH}} \kappa_{\mathrm{S}} \log 2}-\alpha_{\mathrm{RD}} P_{\mathrm{R}}-1\right)}-1\right) \\
P_{\mathrm{R}}=\frac{\left(\alpha_{\mathrm{SR}} P_{\mathrm{S}}+1\right)\left(\left(2 \varepsilon_{\mathrm{SRD}}+\gamma_{\mathrm{SRD}}\right) P_{\mathrm{S}}+2 \alpha_{\mathrm{RD}}\right)}{2\left(\varepsilon_{\mathrm{SRD}} P_{\mathrm{S}}+\alpha_{\mathrm{RD}}\right)\left(\left(\varepsilon_{\mathrm{SRD}}+\gamma_{\mathrm{SRD}}\right) P_{\mathrm{S}}+\alpha_{\mathrm{RD}}\right)} \times \\
\left(\sqrt{1+4 \frac{\left(\varepsilon_{\mathrm{SRD}} P_{\mathrm{S}}+\alpha_{\mathrm{RD}}\right)\left(\left(\varepsilon_{\mathrm{SRD}}+\gamma_{\mathrm{SRD}}\right) P_{\mathrm{S}}+\alpha_{\mathrm{RD}}\right)}{\left(\alpha_{\mathrm{SR}} P_{\mathrm{S}}+1\right)\left(\left(2 \varepsilon_{\mathrm{SRD}}+\gamma_{\mathrm{SRD}}\right) P_{\mathrm{S}}+2 \alpha_{\mathrm{RD}}\right)^{2}}\left(\frac{\gamma_{\mathrm{SRD}} P_{\mathrm{S}}}{q_{\mathrm{TH}} \kappa_{\mathrm{R}} \log 2}-\alpha_{\mathrm{SR}} P_{\mathrm{S}}-1\right)}-1\right)
\end{aligned}
$$

$$
\begin{array}{cl}
\max _{P_{\mathrm{S}}, P_{\mathrm{R}}} & f_{\mathrm{CT}}\left(P_{\mathrm{S}}, P_{\mathrm{R}}\right) \\
\text { subject to } & 0<P_{\mathrm{S}} \leq P_{\mathrm{S}, \max } \\
& 0<P_{\mathrm{R}} \leq P_{\mathrm{R}, \max },
\end{array}
$$

where $f_{\mathrm{CT}}\left(P_{\mathrm{S}}, P_{\mathrm{R}}\right)$ is defined as

$$
\begin{aligned}
f_{\mathrm{CT}}=\left(\frac{1}{\log 2}\right) \log (1 & +\frac{\gamma_{\mathrm{SD}} P_{\mathrm{S}}}{\varepsilon_{\mathrm{SD}} P_{\mathrm{S}}+1} \\
& \left.+\frac{\gamma_{\mathrm{SRD}} P_{\mathrm{S}} P_{\mathrm{R}}}{\varepsilon_{\mathrm{SRD}} P_{\mathrm{S}} P_{\mathrm{R}}+\alpha_{\mathrm{SR}} P_{\mathrm{S}}+\alpha_{\mathrm{RD}} P_{\mathrm{R}}+1}\right) \\
& -q_{\mathrm{CT}}\left(\kappa_{\mathrm{S}} P_{\mathrm{S}}+\kappa_{\mathrm{R}} P_{\mathrm{R}}+2 P_{\mathrm{c}, \mathrm{CT}}+2 P_{\mathrm{CE}}\right)
\end{aligned}
$$

and $q_{\mathrm{CT}}$ is a constant that is equal to the maximum $\mathrm{EE}$ value of the CT scheme, i.e., $q_{\mathrm{CT}}=\max \eta_{\mathrm{CT}}$. In other words, if $\left(P_{\mathrm{S}}^{*}, P_{\mathrm{R}}^{*}\right)=\arg \max \eta_{\mathrm{CT}}$, then $f_{\mathrm{CT}}\left(P_{\mathrm{S}}^{*}, P_{\mathrm{R}}{ }^{*}\right)=0$ [27]. Let $\mathcal{G}_{\mathrm{CT}}$ denote the argument of the logarithmic term, which can be written as $\mathcal{G}_{\mathrm{CT}}=\mathcal{G}_{\mathrm{DT}}+\mathcal{G}_{\mathrm{TH}}$. Thus, the objective function $f_{\mathrm{CT}}\left(P_{\mathrm{S}}, P_{\mathrm{R}}\right)$ is a concave function in $P_{\mathrm{S}}$ for a fixed $P_{\mathrm{R}}$ and is also concave in $P_{\mathrm{R}}$ for a fixed $P_{\mathrm{S}}$ [28]. First we assume fixed $P_{\mathrm{R}}$, then differentiate $f_{\mathrm{CT}}$ with respect to $P_{\mathrm{S}}$ and equate the derivative to zero and obtain

$$
\begin{gathered}
\frac{\gamma_{\mathrm{SD}}}{\left(\varepsilon_{\mathrm{SD}} P_{\mathrm{S}}+1\right)^{2}}+\frac{\left(\alpha_{\mathrm{RD}} P_{\mathrm{R}}+1\right) \gamma_{\mathrm{SRD}} P_{\mathrm{R}}}{\left(\varepsilon_{\mathrm{SRD}} P_{\mathrm{S}} P_{\mathrm{R}}+\alpha_{\mathrm{SR}} P_{\mathrm{S}}+\alpha_{\mathrm{RD}} P_{\mathrm{R}}+1\right)^{2}}= \\
q_{\mathrm{CT}} \kappa_{\mathrm{S}}(\log 2)\left(1+\frac{\gamma_{\mathrm{SD}} P_{\mathrm{S}}}{\varepsilon_{\mathrm{SD}} P_{\mathrm{S}}+1}\right. \\
\left.\quad+\frac{\gamma_{\mathrm{SRD}} P_{\mathrm{S}} P_{\mathrm{R}}}{\varepsilon_{\mathrm{SRD}} P_{\mathrm{S}} P_{\mathrm{R}}+\alpha_{\mathrm{SR}} P_{\mathrm{S}}+\alpha_{\mathrm{RD}} P_{\mathrm{R}}+1}\right) \cdot \text { (42) }
\end{gathered}
$$

Equation (42) gives a unique solution of $P_{\mathrm{S}}$ since it is the intersection of a decreasing function on its left hand side and an increasing function on its right hand side. Then, we assume fixed $P_{\mathrm{S}}$, differentiate $f_{\mathrm{CT}}$ with respect to $P_{\mathrm{R}}$, equate the result to zero, and obtain

$$
\begin{gathered}
\frac{\left(\alpha_{\mathrm{SR}} P_{\mathrm{S}}+1\right) \gamma_{\mathrm{SRD}} P_{\mathrm{S}}}{\left(\varepsilon_{\mathrm{SRD}} P_{\mathrm{S}} P_{\mathrm{R}}+\alpha_{\mathrm{SR}} P_{\mathrm{S}}+\alpha_{\mathrm{RD}} P_{\mathrm{R}}+1\right)^{2}}=(\log 2) q_{\mathrm{CT}} \kappa_{\mathrm{R}} \times \\
\left(1+\frac{\gamma_{\mathrm{SD}} P_{\mathrm{S}}}{\varepsilon_{\mathrm{SD}} P_{\mathrm{S}}+1}+\frac{\gamma_{\mathrm{SRD}} P_{\mathrm{S}} P_{\mathrm{R}}}{\varepsilon_{\mathrm{SRD}} P_{\mathrm{S}} P_{\mathrm{R}}+\alpha_{\mathrm{SR}} P_{\mathrm{S}}+\alpha_{\mathrm{RD}} P_{\mathrm{R}}+1}\right) .
\end{gathered}
$$

We obtain also a unique solution of $P_{\mathrm{R}}$ from (43) because it represents the intersection of a decreasing function on its left hand size and an increasing function on its right hand side. Based on (42) and (43), we develop Algorithm III that uses the Dinklebach method and calculates iteratively the energyefficient power allocation solution.

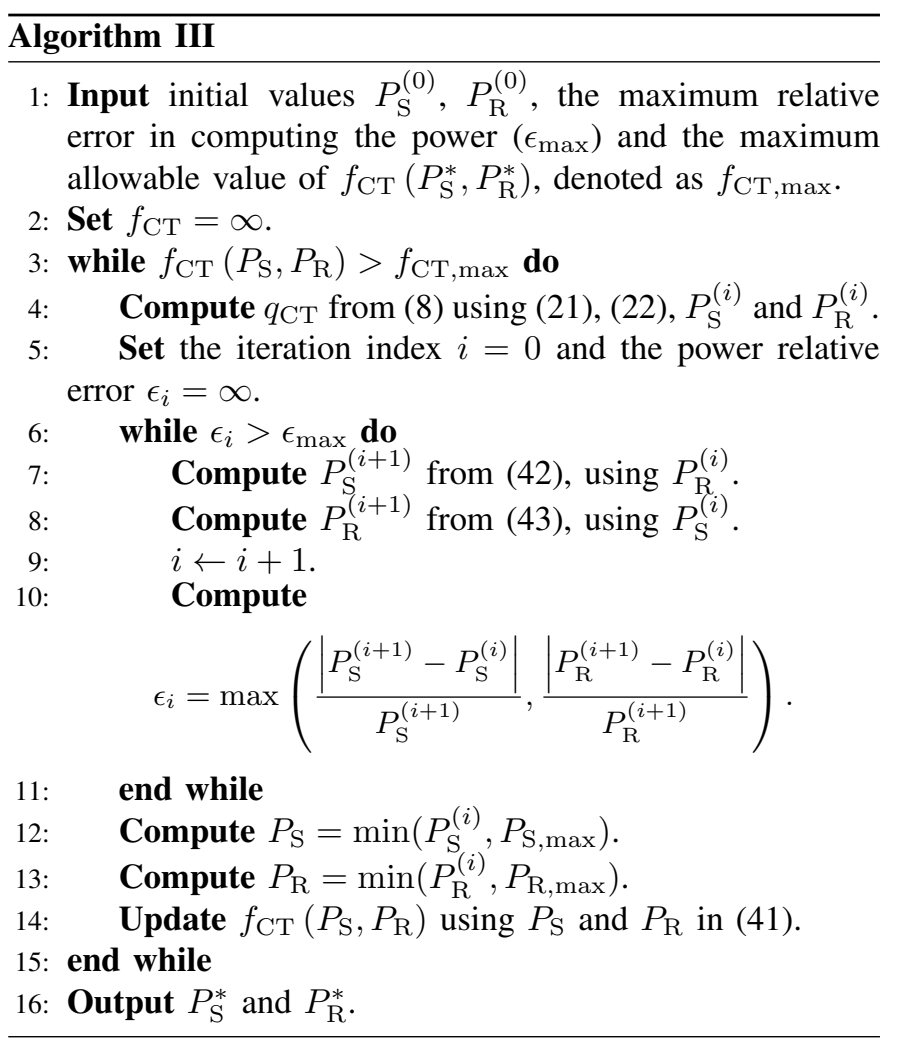

\section{Simulation Results}

In this section, we provide a numerical evaluation of the proposed energy-efficient OAF cooperative system using the two estimation strategies, i.e., CCE and DCE. The circuits power consumption parameters are considered from [29] and listed with other simulation parameters in Table I. The simulation examples use these parameters, unless otherwise specified.

\section{A. Performance Evaluation of the Adaptive Opportunistic Amplify-and-Forward System}

First, we aim to examine the effectiveness of the proposed AOAF system for both strategies versus the distance between 
TABLE I

SiMULATION PARAMETERS.

\begin{tabular}{|c|c|c|}
\hline$P_{\mathrm{ct}, \mathrm{S}}=P_{\mathrm{ct}, \mathrm{R}}=112 \mathrm{~mW}$ & $\kappa_{\mathrm{S}}=\kappa_{\mathrm{R}}=2.88$ & $\nu=3.5$ \\
\hline$P_{\mathrm{S}, \max }=120 \mathrm{~mW}$ & $P_{\mathrm{R}, \max }=120 \mathrm{~mW}$ & $\lambda=0.12 \mathrm{~m}$ \\
\hline$P_{\mathrm{cr}, \mathrm{R}}=P_{\mathrm{cr}, \mathrm{D}}=98 \mathrm{~mW}$ & $d_{\mathrm{SD}}=200 \mathrm{~m}$ & $B=10 \mathrm{kHz}$ \\
\hline$d_{\mathrm{RD}}=0.5 d_{\mathrm{SD}} \mathrm{m}$ & $d_{\mathrm{SR}}=0.5 d_{\mathrm{SD}} \mathrm{m}$ & $d_{0}=1 \mathrm{~m}$ \\
\hline$\sigma_{h_{\mathrm{RD}}}^{2}=\sigma_{h_{\mathrm{SR}}}^{2}=1$ & $N_{\mathrm{f}}=7 \mathrm{~dB}$ & $N=1$ \\
\hline$P_{\mathrm{P}, \mathrm{S}}=P_{\mathrm{P}, \mathrm{R}}=120 \mathrm{~mW}$ & $N_{0}=-171 \mathrm{dBm} / \mathrm{Hz}$ & $\rho=N /(20-N)$ \\
\hline
\end{tabular}

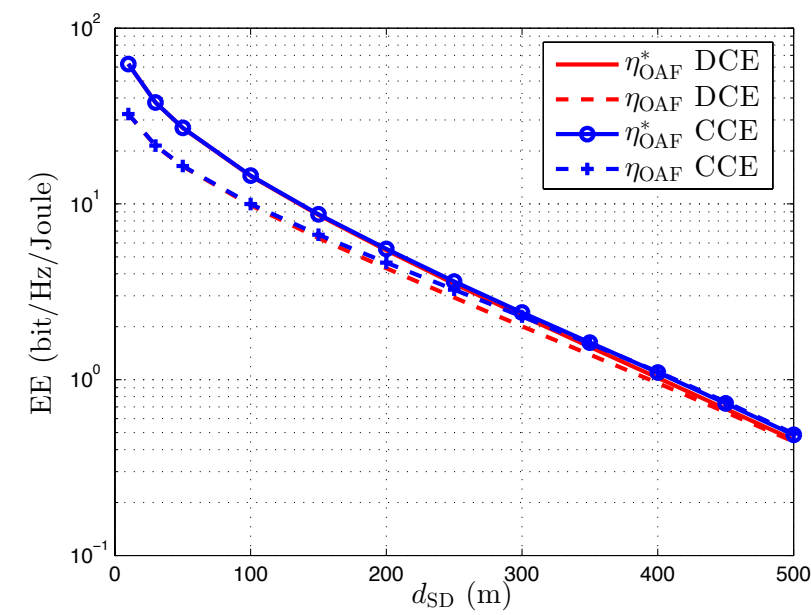

Fig. 2. EE versus the distance between $S$ and $D$ for OAF with maximum power and optimized OAF systems assuming both CCE and DCE strategies.

$S$ and $D$, and the relative relay location through the following three simulation examples.

Simulation Example 1: First, we compare the performance of the proposed energy-efficient OAF with and without power allocation for different estimation strategies. For this purpose, we plot the $\mathrm{EE}$ of the aforementioned systems versus the distance between $S$ and $D$, in Fig. 2, where the OAF is assumed to use maximum power budget. First, we observe that the AOAF system with either CCE or DCE strategies give notable improvement over OAF systems for short-ranges communication. For long-ranges communication, the path loss attenuation increases significantly and the optimized systems tend to use more power for transmission, which tends to reach the maximum power, to guarantee reliable communication. As a result, EE is expected to reduce and $\mathrm{AOAF}$ and $\mathrm{OAF}$ system have similar performance. On the other hand, less power can be used when the channel becomes reliable at short ranges; thus, the gap between the maximum power OAF and optimized OAF systems increases as the distance decreases. Both estimation strategies achieve almost the same performance because they have the same estimation quality [20].

Simulation Example 2: The second simulation example compares the effectiveness of the EE performance of the proposed AOAF and the OAF that uses fixed maximum power versus the relative relay location to the destination. Different estimation strategies are observed to have nearly the same performance for AOAF and OAF, respectively, as shown in Fig. 3. We observe that the maximum EE performance for both OAF

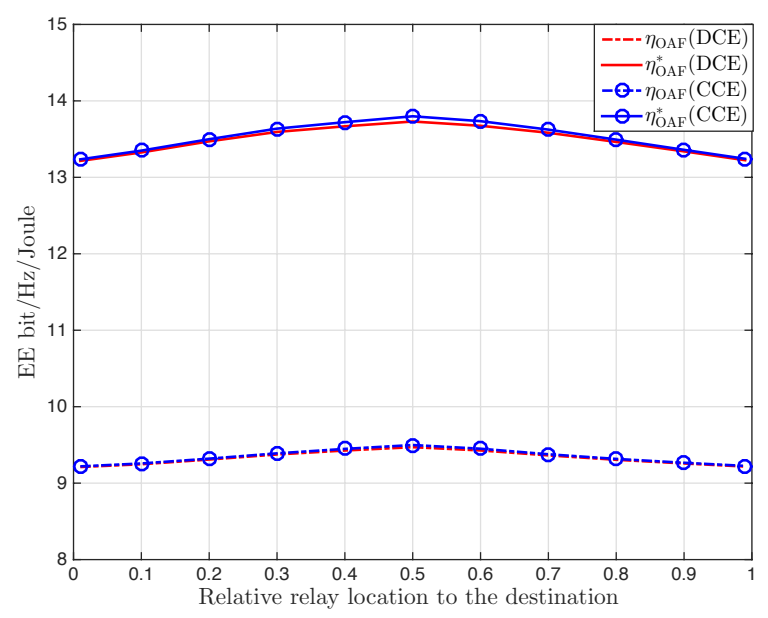

Fig. 3. EE versus the relative $R$ location with respect to $D$ for AOAF ( $\eta_{\mathrm{OAF}}^{*}$ ) and OAF ( $\left.\eta_{\mathrm{OAF}}\right)$ with maximum power usage are both $S$ and $R$ assuming both CCE and DCE strategies.

and AOAF is achieved in the middle relay location between $S$ and $D$, which achieves also the best relative improvement of OAF system. Specifically, when the relay is located near one of the nodes, AOAF achieves $4 \mathrm{bit} / \mathrm{Hz} / \mathrm{Joule}$ improvement over the OAF system. The relative improvement increases reaching a maximum improvement of $4.25 \mathrm{bit} / \mathrm{Hz} / \mathrm{Joule}$ in the middle distance between $S$ and $D$. The observed symmetric behavior is due to the symmetric parameters of both $S$ ans $R$, where asymmetric parameters lead to different performance, as will be seen in the simulation example 6 .

Simulation Example 3: This simulation studies the importance of the channel estimation parameters in formulating the EE metric. Towards this purpose, we compare our proposed AOAF system with the AOAF that allocates the power while neglecting the channel estimation impact on the EE metric. We call the later system "based on perfect CSI design" in Fig. 4, and consider the estimated CSI while imposing a zero value to both the estimation error variances and the consumed power during the estimation phase. From Fig. 4, we observe that taking into account the channel estimation cost is very important for medium and short-ranges communication when the CCE strategy is adopted, while it needs to be considered when the DCE strategy is used. It is worthy mentioning that the DCE strategy is desirable, as it achieves better error probability performance than CCE [20].

\section{B. Contribution of Different Schemes on the Adaptive Oppor- tunistic Amplify-and-Forward System}

Simulation Example 4: In this simulation example, we investigate the maximized EE performance of the DT, TH, and CT schemes when compared to the AOAF system. We assume the usage of the DCE strategy; similar results are obtained using the CCE strategy but not included to avoid redundancy and due to space limitations. Fig. 5 illustrates the relationship between the $\mathrm{EE}$ for each scheme in addition to the AOAF system versus the distance between $S$ and $D$. We observe that DT achieves better EE performance than TH and CT for 


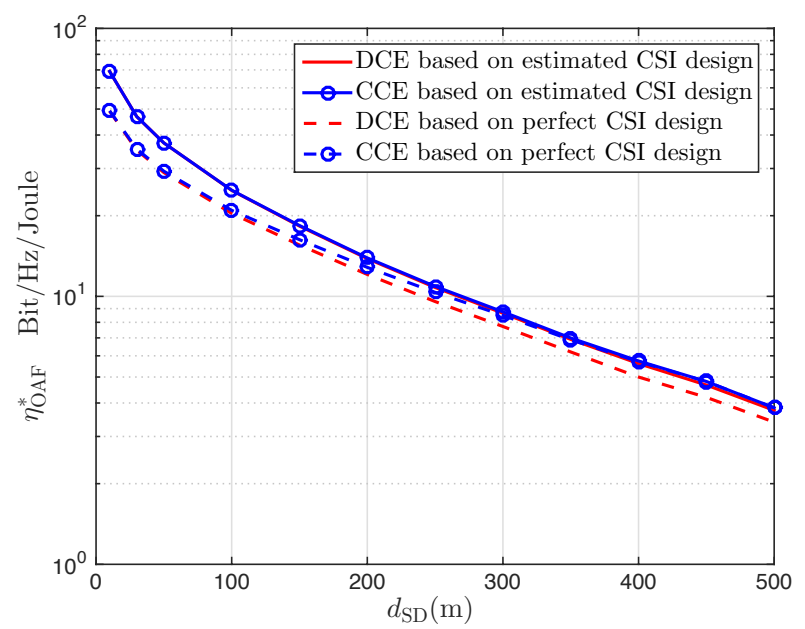

Fig. 4. EE versus the distance between $S$ and $D$ for AOAF ( $\eta_{\mathrm{OAF}}^{*}$ ) based on the proposed impact of estimated CSI and neglecting the impact of CSI estimation.

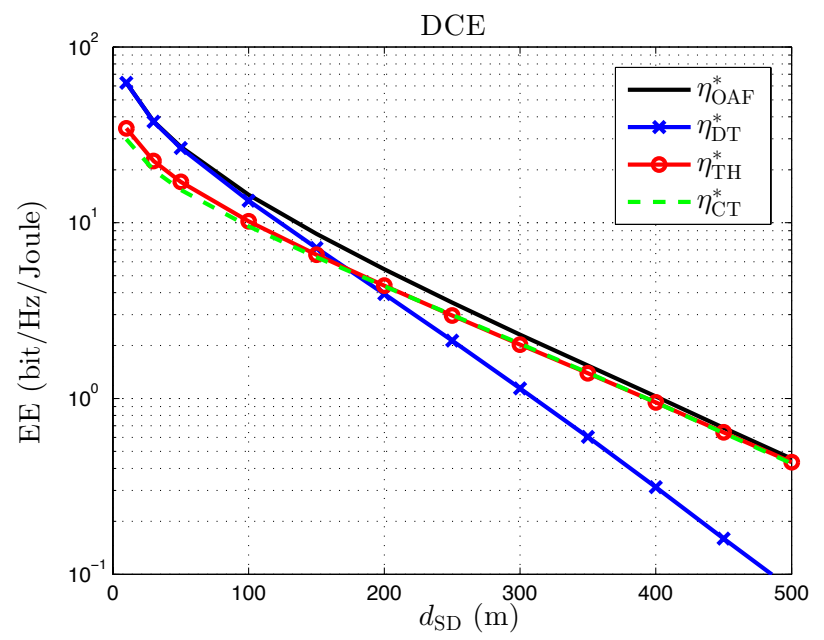

Fig. 5. EE versus the distance between $S$ and $D$ for AOAF and different transmission schemes, DT, TH and CT, assuming DCE strategy.

short-range communications. In this scenario, the direct link channel between $S$ and $D$ is relatively reliable and the required data can be delivered through the direct link without rate loss as in $\mathrm{CT}$ and $\mathrm{TH}$ in addition to saving the energy consumed by the $R$ node and the circuits energy consumption used for reception in the relaying phase at $D$. As the communication range increases, the direct link channel becomes unreliable, as it is subject to notable path loss, which can reduce significantly the SNR and the achievable rate. On the other hand, both the TH and CT schemes get the benefit of the cooperative link and achieve better EE performance when compared with DT scheme at medium and long-range communication.

To investigate the participation ratio for each scheme in the AOAF system, we plot the usage ratio versus the distance between $S$ and $D$ in Fig. 6. As expected from the results discussed in Fig. 5, the DT scheme is mostly chosen for shortrange communications, while for long-range communications, DT become unfavorable, and either TH or CT is chosen in-

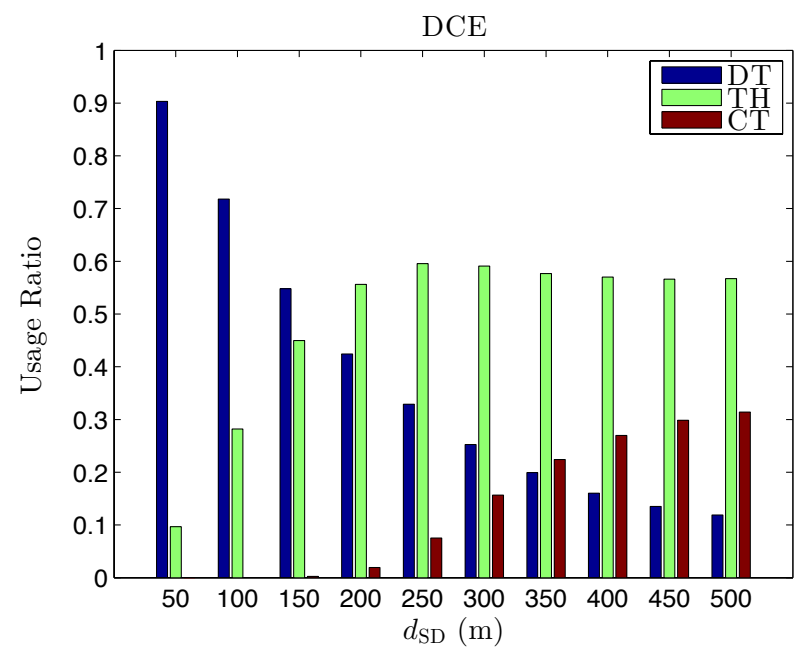

Fig. 6. Usage ratio of different schemes, DT, TH, and CT, versus the distance between $S$ and $D$ assuming the DCE strategy with $P_{\mathrm{cr}, \mathrm{D}}=98 \mathrm{~mW}$.

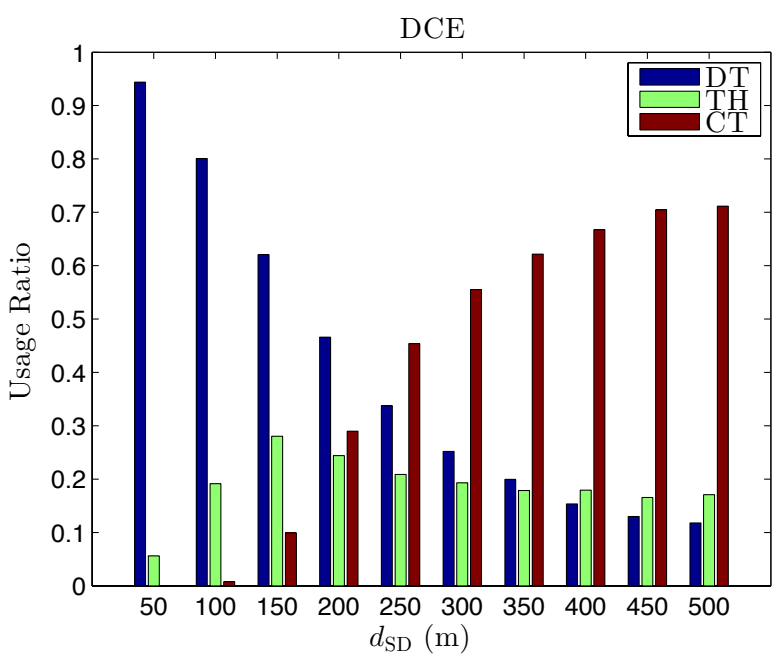

Fig. 7. Usage ratio of different schemes, DT, TH, and CT, versus the distance between $S$ and $D$ assuming the DCE strategy with $P_{\mathrm{cr}, \mathrm{D}}=9.8 \mathrm{~mW}$.

stead. Although the average EE performance of the optimized $\mathrm{TH}$ and CT schemes is almost the same as can be observed in Fig. 5, choosing the best scheme depends significantly on the reception circuits power consumption of $D, P_{\mathrm{cr}, \mathrm{D}}$, whose value in CT is doubled when compared with the TH scheme. To clarify this point, we assume another scenario where $P_{\mathrm{cr}, \mathrm{D}}$ is assumed to have a lower value which equals $9.8 \mathrm{~mW}$. In this second scenario, the EE of CT improves and CT is mostly chosen versus $\mathrm{TH}$ for medium and long-range communications, as can be seen in Fig. 7.

\section{Impact of System Parameters on the Adaptive Opportunis- tic Amplify-and-Forward System}

Simulation Example 5: In this example, we study the effect of the number of pilots $(N)$ and their power on the maximized EE performance of the OAF cooperative systems. In the following simulations we assume symmetrical equal pilots power at both $S$ and $R$ and that the pilots power 


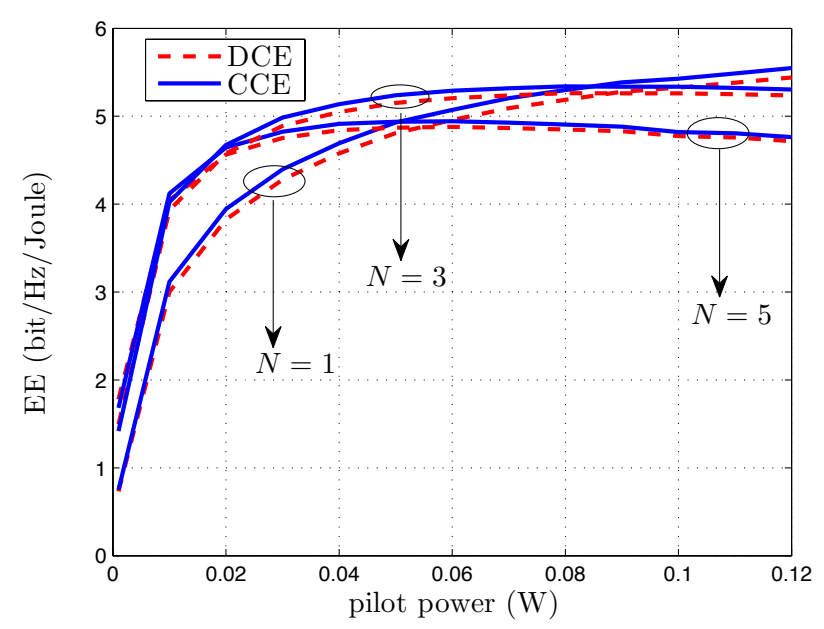

Fig. 8. EE versus the power of pilots assuming different number of pilots for both the CCE and DCE strategies.

changes from $1 \mathrm{~mW}$ to its maximum budget value of $120 \mathrm{~mW}$. Increasing the number of pilots and/or their power is expected to improve the estimation quality for both techniques [20]. In the following, we investigate whether the same performance trend is followed with EE. Fig. 8 shows the EE of AOAF for both the CCE and DCE strategies versus the pilots power assuming different number of pilots $N=1,3$, and 5 . For the single pilot scenario, i.e., $N=1$, we observe that the EE performance improves with the increase of pilots' power, while this trend changes when the number of pilots increases to 3 and 5. Specifically, maximum power-optimized EE can be achieved at $P_{\mathrm{P}, \mathrm{S}}=P_{\mathrm{P}, \mathrm{R}}=80 \mathrm{~mW}$ for $N=3$, and $P_{\mathrm{P}, \mathrm{S}}=P_{\mathrm{P}, \mathrm{R}}=50 \mathrm{~mW}$ for $N=5$. These results indicate that there is also an optimal power value for the single pilot case, but it is expected to be more than the available budget. The existence of the optimal pilot power for maximum $\mathrm{EE}$ means that this power value gives a good channel estimate and the MSE can be considered acceptable in this communication scenario.

Simulation Example 6: Through this example, we aim to study the effect of the relay location on the maximized OAF performance. Fig. 9 plots the EE versus the relative relay location with respect to the destination for three cases that correspond to different maximum power budgets at both $S$ and $R$. Case 1 represents the assumption of symmetrical coefficients, i.e., equal systems parameters at both $S$ and $R$ as listed in Table I with $P_{\mathrm{S}, \max }=P_{\mathrm{R}, \max }=120 \mathrm{~mW}$, while Case 2 is for reduced $S$ power budget with $P_{\mathrm{S}, \max }=$ $12 \mathrm{~mW}$, and Case 3 is for reduced $R$ power budget with $P_{\mathrm{R}, \max }=12 \mathrm{~mW}$. We observe that in Case 1 , the system parameters listed in Table I are symmetric; thus, the EE performance is symmetric versus relay location with maximum performance at the middle location between $S$ and $R$. As the system parameters become asymmetrical, the EE performance becomes also asymmetrical. The optimal $R$ location is near $S$ when the $S$ power budget is less than the $R$, while it is near $D$ when the $R$ power budget is less than for $S$. It is worth noting that the maximum EE performance of Case 2, i.e., with

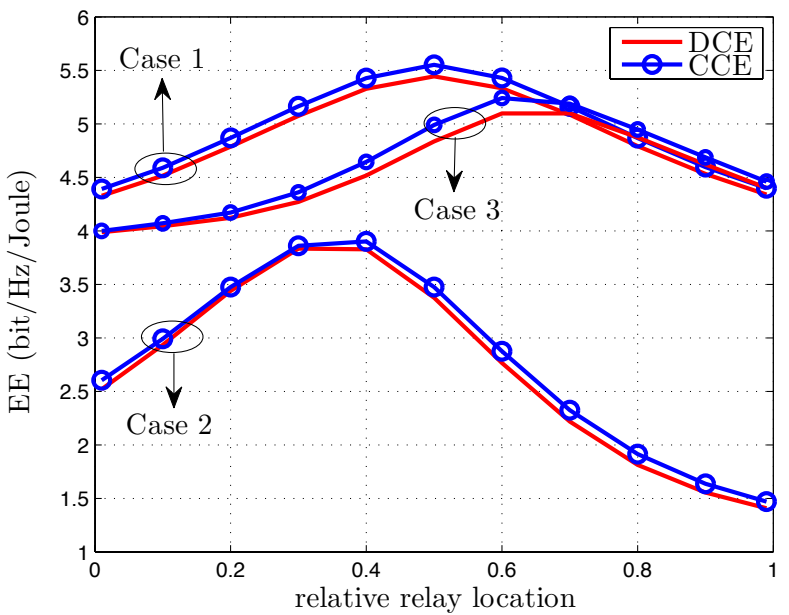

Fig. 9. EE versus the relative relay location with respect to the $S-D$ distance for both the CCE and DCE strategies.

reduced source power budget, is less than its counterpart in Cases 1 and 3. The source power plays an important role in OAF, where it affects mainly the DT scheme; thus the AOAF is expected to have relative degradation when compared with other cases. Both estimation strategies, CCE and DCE, achieve nearly the same EE performance with slight advantages to the CCE strategy.

We observe from the simulation results that the CCE and DCE strategies achieve almost the same performance because both strategies have nearly the same estimation error [20], the power consumption of the estimation phase is also the same and the difference in the SNR becomes negligible due to the logarithmic function that exists in the numerator of the $\mathrm{EE}$ metric.

\section{CONCLUSION}

In this paper, an energy-efficient opportunistic cooperative system is considered to switch between DT, TH, CT schemes in order to achieve maximum EE transmission assuming $\mathrm{AF}$ relaying technique and estimated CSI. Two channel estimation strategies are adopted, namely CCE and DCE, depending on the availability of the channel estimator at the relay node. An accurate EE metric is formulated considering the channel estimation cost in both reducing the SNR and increasing the power consumption due to the insertion of pilots in the transmission packets. An adaptive energy-aware power allocation algorithm is then proposed to maximize the EE metric of the proposed opportunistic cooperative system for both estimation strategies. The proposed OAF system achieves notable energy saving especially for short-range communications. For adaptive power allocation systems, the DT scheme achieves good performance for short-range communications, while both TH and CT achieve better performance than the DT scheme for medium and long-range communications. Improving the channel estimation does not always provide an energy-efficient solution. There exists a certain estimation quality that achieves the maximum $\mathrm{EE}$, where any further improvement in it by 
either increasing number of pilots or their power reduces the available energy budget that should be used for data transmission. Symmetrical source and relay parameters lead to the best relay location in the middle between source and destination, while asymmetrical parameters yield a deviation of the best relay location towards either the source or the destination side. The maximum achievable $\mathrm{EE}$ of the AOAF is controlled mainly by the source parameters.

\section{ACKNOWLEDGMENT}

We would like to thank the editor Dr. Ioannis Krikidis, and the anonymous reviewers for their constructive feedback, which helped improved the quality of the paper.

The work of M. H. Ahmed and O. A. Dobre was supported in part by the Natural Sciences and Engineering Research Council of Canada (NSERC), through its Discovery program. The work of M.-S. Alouini was supported in part by the Qatar National Research Fund (a member of Qatar Foundation) under NPRP Grant NPRP 5-250-2-087. The statements made herein are solely the responsibility of the authors.

\section{REFERENCES}

[1] A. Fehske, G. Fettweis, J. Malmodin, and G. Biczok, "The global footprint of mobile communications: The ecological and economic perspective," IEEE Commun. Mag., vol. 49, no. 8, pp. 55-62, Aug. 2011.

[2] L. M. Correia, D. Zeller, O. Blume, D. Ferling, Y. Jading, I. Gódor, G. Auer, and L. Van Der Perre, "Challenges and enabling technologies for energy aware mobile radio networks," IEEE Commun. Mag., vol. 48, no. 11, pp. 66-72, Nov. 2010.

[3] C. Han, T. Harrold, S. Armour, I. Krikidis, S. Videv, P. M. Grant, H. Haas, J. S. Thompson, I. Ku, C.-X. Wang, T. A. Le, M. R. Nakhai, J. Zhang, and L. Hanzo, "Green radio: Radio techniques to enable energy-efficient wireless networks," IEEE Commun. Mag., vol. 49, no. 6, pp. 46-54, May 2011.

[4] D. Feng, C. Jiang, G. Lim, L. J. Cimini Jr, G. Feng, and G. Y. Li, "A survey of energy-efficient wireless communications," IEEE Commun. Surveys Tutorials, vol. 15, no. 1, pp. 167-178, First Quarter 2013.

[5] A. Nosratinia, T. E. Hunter, and A. Hedayat, "Cooperative communication in wireless networks," IEEE Commun. Mag., vol. 42, no. 10, pp. 74-80, Oct. 2004.

[6] O. Amin, S. Bavarian, and L. Lampe, "Cooperative techniques for energy-efficient wireless communications," in Green Radio Communication Networks. Cambridge University Press, 2012, pp. 125-149.

[7] O. Waqar, M. Imran, M. Dianati, and R. Tafazolli, "Energy consumption analysis and optimization of BER-constrained amplify-and-forward relay networks," IEEE Trans. Veh. Technol., vol. 63, no. 3, pp. 1256-1269, Mar. 2014.

[8] S. Huang, H. Chen, J. Cai, and F. Zhao, "Energy efficiency and spectralefficiency tradeoff in amplify-and-forward relay networks," IEEE Trans. Veh. Technol., vol. 62, no. 9, pp. 4366-4378, Nov. 2013.

[9] C. Sun, Y. Cen, and C. Yang, "Energy efficient OFDM relay systems," IEEE Trans. Commun., vol. 61, no. 5, pp. 1797-1809, May 2013.

[10] K. T. K. Cheung, S. Yang, and L. Hanzo, "Achieving maximum energy-efficiency in multi-relay OFDMA cellular networks: A fractional programming approach," IEEE Trans. Commun., vol. 61, no. 7, pp. 2746-2757, Jul. 2013

[11] R. A. Loodaricheh, S. Mallick, and V. Bhargava, "Energy efficient resource allocation for OFDMA cellular networks with user cooperation and QoS provisioning," vol. 13, no. 11, pp. 6132-6146, Nov 2014.

[12] A. Zappone, P. Cao, and E. Jorswieck, "Low-complexity energy efficiency optimization with statistical CSI in two-hop MIMO systems," IEEE Signal Process. Lett., vol. 21, no. 11, Nov. 2014.

[13] — "Energy efficiency optimization in relay-assisted MIMO systems with perfect and statistical CSI," IEEE Trans. Signal Process., vol. 2, no. 62, pp. 443-457, Jan. 2014.

[14] V.-A. Le, R.-A. Pitaval, S. D. Blostein, T. Riihonen, and R. Wichman, "One-bit CSI feedback selection schemes for energy-efficient multiuser and multirelay systems," IEEE Trans. Wireless Commun., vol. 12, no. 3, pp. 1149-1161, Mar. 2013.
[15] Z. Peng, W. Xu, L.-C. Wang, and C. Zhao, "Achievable rate analysis and feedback design for multiuser MIMO relay with imperfect CSI," IEEE Trans. Wireless Commun., vol. 13, no. 2, pp. 780-793, Feb. 2014.

[16] O. Amin, E. Bedeer, M. H. Ahmed, and O. A. Dobre, "A novel energy efficient scheme with a finite-rate feedback channel," IEEE Wireless Commun. Lett., vol. 3, no. 5, pp. 497-500, Oct. 2014.

[17] O. Amin, S. S. Ikki, and M. Uysal, "On the performance analysis of multirelay cooperative diversity systems with channel estimation errors," vol. 60, no. 5, pp. 2050-2059, Jun. 2011.

[18] S. S. Ikki and S. A'issa, "Two-way amplify-and-forward relaying with Gaussian imperfect channel estimations," IEEE Commun. Lett., vol. 16 no. 7, pp. 956-959, Jul. 2012.

[19] V. Erceg, L. J. Greenstein, S. Y. Tjandra, S. R. Parkoff, A. Gupta, B. Kulic, A. A. Julius, and R. Bianchi, "An empirically based path loss model for wireless channels in suburban environments," IEEE $J$. Sel. Areas Commun., vol. 17, no. 7, pp. 1205-1211, Jul. 1999.

[20] O. Amin, B. Gedik, and M. Uysal, "Channel estimation for amplifyand-forward relaying: Cascaded against disintegrated estimators," IET Commun., vol. 4, no. 10, pp. 1207-1216, Jul. 2010.

[21] H. Mheidat and M. Uysal, "Impact of receive diversity on the performance of amplify-and-forward relaying under APS and IPS power constraints," IEEE Commun. Lett., vol. 10, no. 6, pp. 468-470, Jun. 2006.

[22] S. M. Kay, Fundamentals of Statistical Signal Processing: Estimation Theory. Prentice-Hall, New Jersey, 1993.

[23] E. Biglieri, J. Proakis, and S. Shamai, "Fading channels: Informationtheoretic and communications aspects," IEEE Trans. Inf. Theory, vol. 44, no. 6, pp. 2619-2692, Oct. 1998 .

[24] T. Yoo and A. Goldsmith, "Capacity and power allocation for fading mimo channels with channel estimation error," IEEE Trans. Inf. Theory, vol. 52, no. 5, pp. 2203-2214, May 2006.

[25] S. Cui, A. J. Goldsmith, and A. Bahai, "Energy-constrained modulation optimization," IEEE Trans. Wireless Commun., vol. 4, no. 5, pp. 2349 2360, Sep. 2005.

[26] A. Cambini and L. Martein, Generalized Convexity and Optimization: Theory and Applications. Springer, 2008, vol. 616.

[27] W. Dinkelbach, "On nonlinear fractional programming," Management Science, vol. 13, no. 7, pp. 492-498, Mar. 1967.

[28] S. P. Boyd and L. Vandenberghe, Convex Optimization. Cambridge University Press, 2004.

[29] O. Amin and L. Lampe, "Opportunistic energy efficient cooperative communication," IEEE Wireless Commun. Lett., vol. 1, no. 5, pp. 412 415, Oct. 2012.

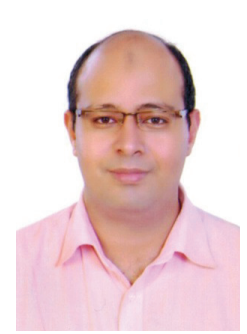

Osama Amin (S'07, M'11, SM'15) received B.Sc. degree in Electrical and Electronics Engineering from Aswan University, Aswan, Egypt, in 2000, M.Sc. degree in Electrical Engineering from Assiut University, Assiut, Egypt in 2004 and Ph.D. degree in Electrical and Computer Engineering, University of Waterloo, Canada in 2010. In June 2012, he joined Assiut University as an Assistant Professor in the Electrical and Electronics Engineering department. Currently, he is with King Abdullah University of Science and Technology (KAUST), Thuwal, Makkah, Kingdom of Saudi Arabia. His general research interests lie in communications systems and signal processing for communications with special emphasis on wireless applications. Specific research areas include green communications, cognitive radio, cooperative communications, and channel estimation. He served as a technical program committee (TPC) member for IEEE VTC, CROWNCOM, PIMRC, and ISSPIT conferences. $\mathrm{He}$ is a co-organizer and a co-chair of the Next Generation Green ICT and 5G Networking (GreeNets) 2015 in the IEEE International Conference on Ubiquitous Wireless Broadband (ICUWB) in Montreal, Canada. 


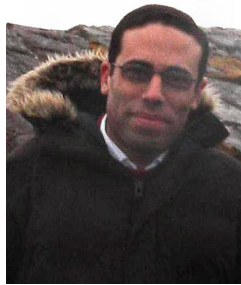

Ebrahim Bedeer received the B.Sc. and M.Sc. degrees from Tanta University, Tanta, Egypt, in 2002 and 2008, respectively and the Ph.D. degree from Memorial University, St. Johns, NL, Canada in 2014, all in Electrical Engineering. Currently, he is a postdoctoral fellow at University of British Columbia, Okanagan campus, Kelowna, BC, Canada. His current research interests are in the area of wireless communications, with a focus on dynamic resource allocation, cognitive radio networks, and green communications.



Mohamed Hossam Ahmed received the B.Sc and M.Sc. degrees in electronics and communications engineering from Ain Shams University, Cairo, Egypt in 1990 and 1994, respectively. He obtained the Ph.D. degree in Electrical Engineering in 2001 from Carleton University, Ottawa, where he worked from 2001 to 2003 as a senior research associate. In 2003, he joined the Faculty of Engineering and Applied Science, Memorial University where he works currently as an Associate Professor. Dr. Ahmed published more than 100 papers in international firstclass journals and conferences. He serves as an Editor for IEEE Communication Surveys and Tutorials and for EURASIP Journal on Wireless Communications and Networking (JWCN), and as an Associate Editor for Wiley International Journal of Communication Systems and Wiley Communication and Mobile Computing (WCMC). He served as a Guest Editor of a special issue on Fairness of Radio Resource Allocation, EURASIP JWCN in 2009, and as a Guest Editor of a special issue on Radio Resource Management in Wireless Internet, Wiley Wireless and Mobile Computing Journal, 2003. Dr. Ahmed is a Senior Member of the IEEE. He serves as a cochair of the Signal Processing Track in ISSPIT' 14 and served as a cochair of the Transmission Technologies Track in VTC'10-Fall, and the multimedia and signal processing symposium in CCECE'09. Dr. Ahmed won the Ontario Graduate Scholarship for Science and Technology in 1997, the Ontario Graduate Scholarship in 1998, 1999, and 2000, and the Communication and Information Technology Ontario (CITO) graduate award in 2000. His research interests include radio resource management in wireless networks, multi-hop relaying, cooperative communication, vehicular ad-hoc networks, cognitive radio networks, and wireless sensor networks. Dr. Ahmed's research is sponsored by NSERC, CFI, Bell/Aliant and other governmental and industrial agencies. Dr. Ahmed is a registered Professional Engineer (P.Eng.) in the province of Newfoundland Canada.

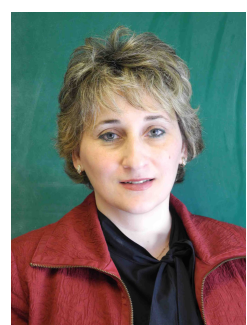

Octavia A. Dobre received the Dipl. Ing. and $\mathrm{Ph}$. D. degrees in ECE from the Polytechnic University of Bucharest (formerly the Polytechnic Institute of Bucharest), Romania, in 1991 and 2000, respectively. In 2000 she was the recipient of a Royal Society scholarship at Westminster University, UK, and in 2001 she held a Fulbright fellowship at Stevens Institute of Technology, USA. Currently, she is an Associate Professor with Memorial University, Canada.

Her research interests include blind signal identification and parameter estimation techniques, cognitive radio systems, transceiver optimization algorithms, dynamic spectrum access, cooperative communications, resource allocation, and coherent optical communications. She published over 150 journal and conference papers in these areas. Dr. Dobre's research has been supported by the Natural Sciences and Engineering Research Council of Canada, Canada Foundation for Innovation, Research and Development Corporation, Defence and Research Development Canada, Communications Research Centre Canada, Altera Corporation, DTA Systems, and ThinkRF among others.

Dr. Dobre is a Senior Editor for the IEEE Communications Letters, as well as an Editor for the IEEE Transactions on Wireless Communications and IEEE Communications Surveys and Tutorials. She has served as an Editor and Guest Editor for several other prestigious journals. She is the General Chair of IEEE CWIT 2015, and has served as Technical Program Co-Chair of symposia at several conferences, including IEEE ICC and GLOBECOM. She is a registered Professional Engineer (P. Eng.) in the province of Newfoundland, Canada.

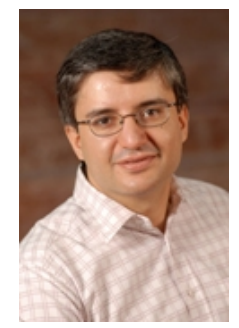

Mohamed-Slim Alouini (S'94, M'98, SM'03, F'09) was born in Tunis, Tunisia. He received the Ph.D. degree in Electrical Engineering from the California Institute of Technology (Caltech), Pasadena, CA, USA, in 1998. He served as a faculty member in the University of Minnesota, Minneapolis, MN, USA, then in the Texas A\&M University at Qatar, Education City, Doha, Qatar before joining King Abdullah University of Science and Technology (KAUST), Thuwal, Makkah Province, Saudi Arabia as a Professor of Electrical Engineering in 2009. His current research interests include the modeling, design, and performance analysis of wireless communication systems. 